\title{
Percepción diferencial de la intervención para la convivencia entre escolares de Santo Domingo, República Dominicana
}

\section{Differential Perception of Intervention for coexistence among school children in Santo Domingo, Dominican Republic}

\author{
Carolina Bringas-Molleda* \\ Josefina Mercedes Acosta** \\ David Álvarez-García*** \\ Margarita Almonte-Mata** \\ Francisco Javier Rodríguez-Díaz*** \\ *Universidad de Extremadura, España \\ **Universidad Autónoma de Santo Domingo, República Dominicana \\ ***Universidad de Oviedo, España
}

\section{Resumen}

La convivencia escolar refiere una de las dificultades a resolver en el ámbito académico. El estudio plantea analizar las propiedades psicométricas de la escala M-EP, adaptado a la República Dominicana, investigar la percepción de normas de convivencia escolar por parte de los estudiantes de la comunidad educativa de Santo Domingo, y su influencia en la aparición de situaciones violentas dentro de marco escolar. Participaron 1945 estudiantes procedentes de centros educativos de Santo Domingo, de edades comprendidas entre 11 y 17 años. Los instrumentos utilizados fueron: El M-EP, que evalúa medidas de mejora de la convivencia escolar percibida, y el CUVE3-EP, que evalúa la frecuencia con la que el alumnado considera que ocurren diferentes tipos de violencia escolar. Los resultados se discuten con el objeto de orientar una intervención más eficaz.

Palabras clave: Violencia escolar, Convivencia, Percepción, Adolescentes, Pautas comportamentales.

\section{Abstract}

School connivance is referred to as one of the difficulties to be solved in the academic field. The study proposes to analyze the psychometric properties of the M-EP scale, adapted to the Dominican Republic and to investigate the perception of norms of school connivance by students from the educational community of Santo Sunday and its influence in the appearance of violent situations in the school setting. 1945 students from different primary education centers in Santo Domingo participated, aged between 11 and 17 years old. The instruments used were: The M-EP, which evaluates measures to improve school connivance perceived, and, the CUVE3-EP, which assesses the frequency with which the students consider that occur different types of school violence. The results are discussed in order to guide a more effective intervention.

Keywords: School violence, Connivance, Perception, Adolescents, Behavioral patterns. 


\section{INTRODUCCIÓN}

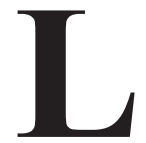

a convivencia escolar se puede manifestar de maneras diversas en el contexto escolar, siendo la aparición de situaciones violentas la que en la actualidad preocupa más a la sociedad. En algunos casos se caracterizan por ser comportamientos disruptivos dentro de su contexto, más que agresivos en relación con el otro (Álvarez-García, Mercedes, Rodríguez, y Núñez, 2015; Pérez-Fuentes, Gázquez, Fernández-Baena, y Molero, 2011). Desde esta realidad se observa que las actitudes hacia el bullying han cambiado en las últimas décadas: aún no hace mucho tiempo era común escuchar que la intimidación era una parte natural del crecimiento, algo que deberías aprender a hacer frente. Sin embargo, además de comportamientos físicos, verbales o psicológicos contra otros, hoy los análisis tienden a destacar los comportamientos disruptivos dentro del contexto escolar (Álvarez-García, Dobarro, Álvarez-Pérez, Núñez, y Rodríguez, 2014).

La violencia escolar como temática ha cobrado relevancia en la comunidad científica en los últimos años (Hymel, y Swearer, 2015; Postigo, González, Montoya, y Ordóñez, 2013; Postigo, Schoeps, Ordóñez, y Montoya-Castilla, 2019; Smith, y López-Castro, 2017), y concretamente el acoso entre iguales preocupa a la sociedad actual, cuya concienciación viene dada principalmente por los medios de comunicación, que únicamente informan de casos graves de suicidio u hospitalización. Se asume que la violencia en el marco escolar se puede prevenir en función del conocimiento que se tenga del contexto, el cual ofrecería las oportunidades para realizar tales comportamientos.

Uno de los entornos más importantes en el desarrollo de una persona, por el tiempo de convivencia con los demás, es la escuela (Compton, Campbell, y Mergler, 2014; Eljach, 2011; Veenstra, Lindenberg, Huitsing, Sainio, y Salmivalli, 2014). En ella cabe resaltar que algunas conductas pueden ser consideradas agresiones por algunas personas y no por otras, dependiendo de su contexto interpretativo, donde el fenómeno del bullying se entiende hoy como un comportamiento intencional y reiterado entre iguales, de carácter agresivo, adoptando el papel de víctima, agresor y/o testigo (Olweus, 2012; Smith, Kwak, y Toda, 2016).

Ello supone analizar la calidad de las relaciones y las oportunidades para ordenar el proceso de aprendizaje conductual, cognitivo e interpersonal de habilidades significativas en su medio para dar respuestas adaptadas a las exigencias de éste (López-Latorre, Garrido, Rodríguez, y Paíno, 2002). Es 
decir, en qué medida se facilitan unas óptimas condiciones de aprendizaje (Novo, Herbón, y Amado, 2016) y marca la calidad en educación para la convivencia, al considerar el papel esencial de la escuela en la adaptación del niño a la sociedad, en la enseñanza de conocimiento y valores, actitudes y hábitos, así como en la promoción de comportamientos de protección ante situaciones de riesgo de violencia y victimización (Dick, y Ferguson, 2015; Peñalva-Vélez, López-Goñi, Vega-Osés, y Satrústegui-Azpíroz, C., 2015).

La realidad de la convivencia escolar por la investigación actual, viene señalando variaciones considerables entre los países en cuanto a la prevalencia y la naturaleza de la victimización (Smith, y Robinson, 2019). Los datos refieren una prevalencia de victimización muy elevada, situándose entre 50 y 70 por ciento de la población estudiantil en América Latina y el Caribe (Eljach, 2011). Los escasos estudios sistemáticos y específicos publicados sobre la violencia escolar en la República Dominicana (Mercedes, 2016; Pacheco-Salazar, 2018; Pacheco-Salazar, y Hernández-Valerio, 2014; Román, y Murillo, 2011; Vargas, 2010) constatan una fuerte presencia (se alcanzan cifras en Primaria de 60 por ciento de víctimas de violencia en el último mes, siendo más de 40 por ciento de robos, aproximadamente 30 por ciento de insulto o amenaza y más de 20 por ciento físico). Estos porcentajes aumentan cuando se pregunta al alumnado si conocen a alguien de su aula que haya sido víctima de algún tipo de actos violentos en la escuela en el último mes.

Si el ámbito escolar es importante, las relaciones en el ámbito familiar de los niños y adolescentes con sus padres deben ser resaltadas con el mismo valor. Se ha demostrado que los estilos parentales influyen en la socialización de los menores y, por ende, en el desarrollo de conductas (Ruiz-Hernández, Moral-Zafra, Llor-Esteban y Jiménez-Barbero, 2019). Ello nos permitirá asumir medidas de prevención temprana, al mismo tiempo que cuidar la relación que mantienen con el grupo de iguales, sabiendo de la influencia de los amigos en el desarrollo de conductas antisociales (Borowsky, Taliaferro, y McMorris, 2013; Cutrín, Gómez-Fraguela, Maneiro, y Sobral, 2017; McGee, Logan, Samuel, y Nunn, 2017; Alarcón, Pérez, Wenger, Salvo y Chesta, 2018).

Los estilos relacionales en los centros ofrecen otra medida del grado de convivencia con la autoridad, que viene a sustituir en estas edades la influencia de la propia familia. Nos referimos a la convivencia escolar que deriva en diferentes tipos de violencia escolar protagonizados por el alumnado y el profesorado, y que resulte percibida por los alumnos. Aquí 
resaltamos las conductas disruptivas (aquellas que obstaculizan el proceso de enseñanza-aprendizaje); aquellas de índole verbal del alumnado hacia otros compañeros o el profesor (el trato improcedente); las propias de violencia física directa o indirecta por parte del alumnado; y también las de rechazo o exclusión social de los compañeros o del profesor hacia el alumno (Álvarez-García, Nuñez y Dobarro, 2013). Ello lleva anexionado la necesidad de evaluar las medidas que se desarrollan para el logro de una mejor convivencia en el centro, así como la propia percepción de sus integrantes. El objeto de estas medidas sería el generar condiciones para mejorar el proceso de enseñanza-aprendizaje por parte del alumnado, al mismo tiempo que se trata de dar respuesta a valores presentes en la resolución de conflictos (cuando se fomenta la ayuda como convivencia) a través del consenso de normas en el aula, tanto para la elaboración como la difusión al alumnado o la familia. El consenso de normas se ha relacionado con un menor comportamiento conflictivo dentro del aula y un decremento de violencia verbal ejercida por los alumnos y profesores. Ello confirma que determinados métodos disciplinarios como el castigo son cuanto menos discutibles (Álvarez-García, Dobarro, Rodríguez, Núñez, y Álvarez, 2013; Yeager, Fong, Lee, y Espelage, 2015).

Ante la complejidad de los factores asociados con la violencia percibida por el alumno y las medidas para el mejor desarrollo de la convivencia en el marco escolar, la investigación asume la comunidad educativa como un concepto amplio donde se implican todos los agentes de socialización, manteniendo un enfoque colaborativo. La conexión de los adolescentes con la escuela es fundamental para reducir e inhibir la conducta violenta, incrementando la motivación en función de creencias, percepciones y expectativas de la familia y profesorado, así como logrando una mejoría en el rendimiento escolar (Smith y López-Castro, 2017; Smith y Robinson, 2019; Méndez y Cerezo, 2018). Este panorama orienta a plantear como objetivos del estudio: analizar las propiedades psicométricas del cuestionario (M-EP) adaptándolo a la República Dominicana, para proporcionar un instrumento que evalúe la percepción del alumnado en Educación Primaria de las medidas de convivencia desarrolladas en el marco escolar; igualmente, se pretende examinar la percepción del nivel de convivencia escolar por estudiantes en la comunidad educativa de la ciudad de Santo Domingo (República Dominicana), teniendo en cuenta la influencia diferencial de variables personales y educativas. Finalmente, en función de la percepción identificada, se pretende conocer la influencia que las normas de conviven- 
cia tienen en la aparición de situaciones violentas dentro de marco escolar de la República Dominicana.

\section{Método}

\section{Participantes}

La muestra fue conformada aleatoriamente por 1,945 estudiantes del segundo ciclo del nivel básico, procedentes de 14 centros educativos diferentes de Santo Domingo (República Dominicana), siendo la procedencia de centros situados en zonas urbanas de 890 alumnos (45.8 por ciento) y los 1,055 restantes ( 54.2 por ciento) de zonas marginales. Por sexos, son 928 varones (47.7 por ciento) y 1017 mujeres (52.3 por ciento). Las edades abarcan el rango entre los 11 y 17 años $(\mathrm{M}=12.71$; DT $=1.15)$. A nivel académico, los participantes se encuentran en los cursos entre $5^{\circ}$ y $8^{\circ}$ grado. Los tres primeros grados, $5^{\circ}, 6^{\circ}$ y $7^{\circ}$ abarcan la mayor proporción de los estudiantes con edades comprendidas entre los 11 y 13 años $(\mathrm{N}=141 ; 98.6$ por ciento; $\mathrm{N}=336 ; 91.8$ por ciento; $\mathrm{N}=500 ; 91.2$ por ciento respectivamente), en tanto que entre los que se encuentran en último curso, existe una mayor variabilidad: 560 alumnos de 11 a 13 años (63.1 por ciento), frente a 328 (36.9 por ciento), quienes tienen entre 14 y 17 años.

\section{Instrumentos de evaluación y variables}

En primer lugar, mediante una prueba ad hoc se ha recogido información sobre los datos personales de los participantes, tales como edad, sexo, curso académico, centro de estudios, así como cuestiones relativas a su entorno familiar y su dedicación al ámbito académico fuera del horario escolar, o actividades de tiempo libre. A continuación, se pasó el Cuestionario M-EP (validado en población española por Álvarez-García, Núñez, Dobarro, y Rodríguez, 2012), para evaluar medidas de mejora de la convivencia escolar percibida por los alumnos. El Cuestionario consta de 18 items en formato de respuesta tipo Likert de cuatro alternativas, que ofrecen el grado de acuerdo o desacuerdo con cuatro opciones de respuesta, desde 1 (Totalmente en desacuerdo hasta) 4 (Totalmente de acuerdo). La estructura del cuestionario se ve conformada por cuatro factores: Educación en Valores y Resolución de Conflictos (EVRC), refiere el fomento de la convivencia y la ayuda mutua (cuando hay conflictos entre el alumnado, el profesorado se esfuerza en ayudarles a que lo resuelvan, mediando entre ellos); Consenso de Normas de Aula (CNA), esto es, elaboración de pautas comportamentales dentro del aula (cada cierto tiempo los profesores y los 
alumnos revisan las normas o reglas y su grado de cumplimento); Difusión de Normas y Sanciones a Familia (DNSF), información dada a las familias sobre las normas escolares y consecuencias de su incumplimiento (Las familias han sido informadas de las normas del centro); y Difusión de Normas y Sanciones a Alumnado (DNSA), información dada al alumnado sobre las normas y consecuencias de su incumplimiento (el alumnado conoce las sanciones a aplicar en caso de no respetar las normas de convivencia en el aula). La prueba ofrece un alpha de Cronbach de 0.861 en su aplicación a la población de España.

Finalmente, se les ha facilitado el Cuestionario CUVE3-EP (ÁlvarezGarcía, Núñez, y Dobarro, 2013), adaptada a población dominicana por Álvarez-García et al. (2015), para evaluar la frecuencia con la que el alumnado considera que ocurren diferentes tipos de violencia escolar, protagonizada por los estudiantes y docentes de su grupo/ aula. Consta de 36 items a responder mediante una escala tipo Likert de cinco alternativas, que van desde 1 (Nunca) hasta 5 (Siempre), referidos a diferentes situaciones que ocurren en el entorno escolar. Ofrece una estructura consistente compuesta por siete factores o tipos de violencia escolar (definidos a continuación, con su correspondiente índice de fiabilidad). Disrupción en el aula (DA): Conductas dirigidas a la obstaculización en el proceso de enseñanza-aprendizaje (los alumnos dificultan las explicaciones del profesor con su comportamiento durante la clase) (0.636); Violencia Verbal del Alumnado hacia el Alumnado (VVAA): Trato verbal improcedente entre los propios alumnos (los alumnos le ponen nombres a sus compañeros o compañeras para molestarlos) (0.606); Violencia Verbal del Alumnado hacia el Profesorado (VVAP): Trato verbal improcedente de los alumnos a los docentes (los alumnos hablan mal de los profesores) (0.712); Violencia Física Directa y Amenazas entre Estudiantes (VFDAE): Actos de agresión física y verbal entre los compañeros (los estudiantes dan golpes a compañeros o compañeras dentro de la escuela) (0.750); Violencia Física Indirecta por parte del Alumnado (VFIA): Comportamientos inadecuados dirigidos a objetos pertenecientes a otros compañeros con la intención de hacer daño (algunos estudiantes rompen o dañan a propósito materiales del centro) (0.707); Exclusión Social entre Estudiantes (ES): Conductas de rechazo a otros compañeros por razones varias (algunos estudiantes son rechazados por sus compañeros porque sacan buenas notas) (0.739); Violencia del Profesorado hacia el Alumnado (VPA): Actos de agresión física o verbal que ejerce el profesor hacia los alumnos (los profesores dan gol- 
pes a los alumnos) (0.750). La fiabilidad global de la escala para nuestro estudio ha sido de 0.921

\section{Procedimiento}

Nuestro interés era obtener una muestra representativa en función de la zona en el que se encontraban, por lo que la selección de los centros se ha realizado de manera aleatoria en base a ese criterio. Por un lado, se seleccionó una serie de centros situados en zona urbana-marginal, dentro de los sectores más empobrecidos y donde los alumnos viven en condiciones precarias; estas zonas se caracterizan por encontrarse en riesgo de situaciones conflictivas, como violencia callejera, problemas de tráfico de drogas, existencia de bandas nacionales, o robos y asaltos. Una vez seleccionados los centros, se solicitó la autorización al director del distrito y del centro educativo, explicándoles los objetivos de la investigación. Por otro lado, se pidió la colaboración de centros educativos situados en zona urbana, cuyos estudiantes se identifican por las condiciones y recursos. Previamente, al ser los participantes menores de edad, se pidió consentimiento a los padres o tutores legales, al mismo tiempo que se les explicó el objetivo de la investigación, asegurándoles la confidencialidad de las respuestas a dar a los instrumentos de evaluación. Igualmente, se apuntó a que todos los análisis serían realizados con la muestra poblacional al completo, de forma colectiva.

\section{Análisis de los datos}

Los datos han sido procesados mediante el paquete estadístico SPSS.26, tomando las variables referidas a las características sociodemográficas de los estudiantes: Sexo; edad (se ha agrupado en dos categorías, de 11-13 años y 14-17 años); rendimiento académico, donde se han considerado tres niveles: Los que no han repetido nunca curso, los que han repetido una vez, y los que han repetido dos veces ó más; curso académico, y tipo de centro educativo (Urbano y Urbano-Marginal).

Una vez obtenidos los datos, tomando como punto de partida el análisis factorial exploratorio de la muestra española (Álvarez-García, Núñez, Dobarro, y Rodríguez, 2012), donde se ha obtenido cuatro factores (explicados en el apartado instrumentos), se ha procedido a realizar los análisis descriptivos de los 18 items de los que componen dichos factores y correlación item-total corregida. Seguidamente se ha calculado el índice de fiabilidad para cada uno de los factores, así como de la escala total, a la vez que se ha analizado la correlación entre los cuatro factores. Finalmente, con el 
objetivo de explorar la adaptación de la estructura del instrumento a la población de escolares de los centros educativos de República Dominicana, se ha procedido a realizar el análisis factorial confirmatorio (AMOS), utilizándose los índices de ajuste del modelo el CFI, NFI, RFI, TLI y RMSEA.

Ello ha dado paso, en primer lugar, a realizar un análisis descriptivo para conocer la frecuencia con que se producen actos de violencia escolar dentro del aula. A continuación, se ha comprobado la normalidad de la muestra mediante la prueba de Kolmogorov-Smirnov, donde se ha podido resaltar que no existe distribución normal. A partir de este momento y con el objetivo de conocer la percepción de los estudiantes acerca de las medidas de mejora de la convivencia escolar en función de la edad, sexo, y tipo de centro (urbano y urbano-marginal), se ha realizado un contraste de medias, mediante la prueba no paramétrica de $U$ de Mann-Whitney. En estos casos se ha calculado también el tamaño de efecto, siguiendo las sugerencias de APA (2009) y Wasserstein y Lazar (2016), para cuya interpretación nos basaremos en la propuesta de Cohen (1988). Para las variables relacionadas con el curso académico y rendimiento se ha utilizado la prueba de Kruskal-Wallis, debido a que tiene más de dos niveles de la variable independiente. Por último, con el objetivo de comprobar la influencia que las medidas de convivencia tienen en la posible aparición de situaciones violentas en el ámbito escolar, se ha realizado un análisis de regresión lineal.

\section{Resultados}

La cantidad de datos que se emanan de la respuesta a los objetivos del estudio, nos permite hacer visibles únicamente aquellos que resultan significativos, explicándose el resto en el texto. En primer lugar, el análisis de la percepción del alumnado en Educación Primaria de las medidas de convivencia desarrolladas en el marco escolar de la República Dominicana, nos lleva a realizar el análisis descriptivo de cada uno de los enunciados de los factores que conforman el cuestionario de evaluación de medidas para la convivencia (Tabla 1): se ofrecen la media y desviación típica de cada item, la asimetría y curtosis, y la correlación item-total corregida. Tanto la prueba de Kolmogorov-Smirnov como los índices de índices de asimetría y curtosis obtenidos atestiguan que los enunciados no siguen una distribución normal. Todos los items muestran una asimetría negativa, lo que significa que el alumnado tiende a marcar los valores altos de la escala. 
Tabla 1: Estadísticos descriptivos: Media y Desviación típica, índices de asimetría y curtosis, y correlación item-total corregida 2017-2018

\begin{tabular}{lrrrrrr}
\hline Factores & Items & $\mathrm{X}$ & $\mathrm{DT}$ & $\begin{array}{r}\text { Asim. } \\
(\mathrm{ET}=0.055)\end{array}$ & $\begin{array}{r}\text { Curt. } \\
(\mathrm{ET}=0.11)\end{array}$ & $\mathrm{r}_{\mathrm{i}-\mathrm{t}}$ \\
\hline & 3 & 3.13 & 0.95 & -0.90 & -0.18 & 0.47 \\
Educación en & 7 & 2.98 & 1.00 & -0.69 & -0.59 & 0.51 \\
Valores y & 9 & 2.94 & 1.02 & -0.62 & -0.75 & 0.49 \\
Resolución de & 11 & 3.15 & 0.97 & -0.92 & -0.22 & 0.59 \\
Conflictos (EVRC) & 13 & 2.85 & 1.03 & -0.48 & -0.93 & 0.50 \\
& 14 & 3.06 & 0.96 & -0.79 & -0.32 & 0.59 \\
& 16 & 3.07 & 0.99 & -0.79 & -0.48 & 0.55 \\
& 17 & 3.01 & 0.96 & $0-.72$ & -0.41 & 0.53 \\
Consenso de Normas & 6 & 2.77 & 1.02 & -0.40 & -0.95 & 0.51 \\
en el Aula (CNA) & 10 & 2.92 & 1.00 & -0.61 & -0.68 & 0.50 \\
& 12 & 2.69 & 1.05 & -0.32 & -1.09 & 0.44 \\
Difusión de Normas & 2 & 2.94 & 0.99 & -0.65 & -0.61 & 0.42 \\
y Sanciones a & 5 & 3.18 & 0.93 & -0.98 & 0.05 & 0.53 \\
Alumnado (DNSA) & 15 & 3.09 & 0.95 & -0.83 & -0.27 & 0.60 \\
& 1 & 3.06 & 1.01 & -0.85 & -0.40 & 0.49 \\
Difusión de Normas & 4 & 2.87 & 1.01 & -0.58 & -0.76 & 0.44 \\
y Sanciones a & 8 & 3.05 & 1.01 & -0.78 & -0.51 & 0.55 \\
Familia (DNSF) & 18 & 3.20 & 0.96 & -1.00 & -0.03 & 0.58 \\
\hline
\end{tabular}

Fuente: elaboración propia a partir de los resultados obtenidos en el análisis de datos.

Respecto a la curtosis, los índices son negativos para casi todos los items (excepto para el item 5); ello indica que las puntuaciones se agrupan menos y se sitúan por debajo de la curva de la distribución normal. De igual manera, se puede observar que la correlación del ítem con el total es siempre positiva, situándose los valores comprendidos en el rango entre 0.42 y 0.60 , al mismo tiempo que el análisis de correlaciones entre los factores postulados por el cuestionario adaptado a la población de España (ver Tabla 2) ofrece correlaciones positivas y estadísticamente significativas entre sí. 
Tabla 2: Matriz de correlaciones entre los cuatro factores del Cuestionario de medidas para la convivencia en Educación Primaria 2017-2018

\begin{tabular}{lcccr}
\hline Factores & EVRC & CNA & DNSA & DNSF \\
\hline EVRC & 1 & & & \\
CNA & $0.63^{* *}$ & 1 & & \\
DNSA & $0.65^{* *}$ & $0.47^{* *}$ & 1 & 1 \\
DNSF & $0.66^{* *}$ & $0.48^{* *}$ & $0.63 * *$ & \\
\hline EVRC: Educación en Valores y Resolución de Conflictos; CNA: Consenso de Normas en el Aula; \\
DNSA: Difusión de Normas y Sanciones a Alumnado; DNSF: Difusión de Normas y Sanciones a \\
Familia. \\
**p $<0.01$ \\
Fuente: elaboración propia a partir de los resultados obtenidos en el análisis de datos.
\end{tabular}

Estos resultados dan paso a verificar el ajuste del modelo obtenido en la adaptación a los escolares de España. Se utiliza como propuesta de modelo para el análisis factorial confirmatorio el compuesto por cuatro factores interrelacionados: Difusión de Normas y Sanciones entre las Familias; Difusión de Normas y Sanciones entre el Alumnado; Educación en Valores y Resolución de Conflictos, y Consenso de Normas de Aula. Cada item del cuestionario es explicado únicamente por un factor (Figura 1), revelando los resultados obtenidos que la propuesta del instrumento adaptado ofrece unos índices estadísticos satisfactorios para que sea considerado válido: $\mathrm{CFI}=0.955 ; \mathrm{NFI}=0.942 ; \mathrm{RFI}=0.931$; TLI: 0.946: $\mathrm{RMSEA}=0.041 . \mathrm{El}$ modelo resultante vuelve a referir que las correlaciones entre los cuatro factores son positivas (Figura 1), siendo la consistencia del cuestionario, medida a través del Alpha de Cronbach, elevada (0.88) y aceptable para los factores propuestos (resultados que varían en el rango entre 0.57 y 0.79 ).

A continuación, pretendiendo examinar la percepción de los estudiantes del nivel de convivencia escolar en la comunidad educativa de la ciudad de Santo Domingo, se ofrecen los estadísticos descriptivos que indican la frecuencia de cada uno de los tipos de violencia escolar percibidos en los centros educativos de nuestra población muestral (ver Tabla 3). Los resultados indican que el nivel más bajo ocurre en relación a actos de agresión física o verbal que ejerce el profesor hacia los alumnos, en tanto que los más frecuentes son aquellos referidos a la disrupción en el aula. 
Percepción diferencial de la intervención para la convivencia entre escolares de Santo Domingo ... / C. BRINGAS et al.

Figura 1: Análisis Factorial Confirmatorio de la propuesta del Modelo de Cuatro factores de los 18 enunciados del Cuestionario medidas para la convivencia en Educación Primaria (M-EP) 2017-2018

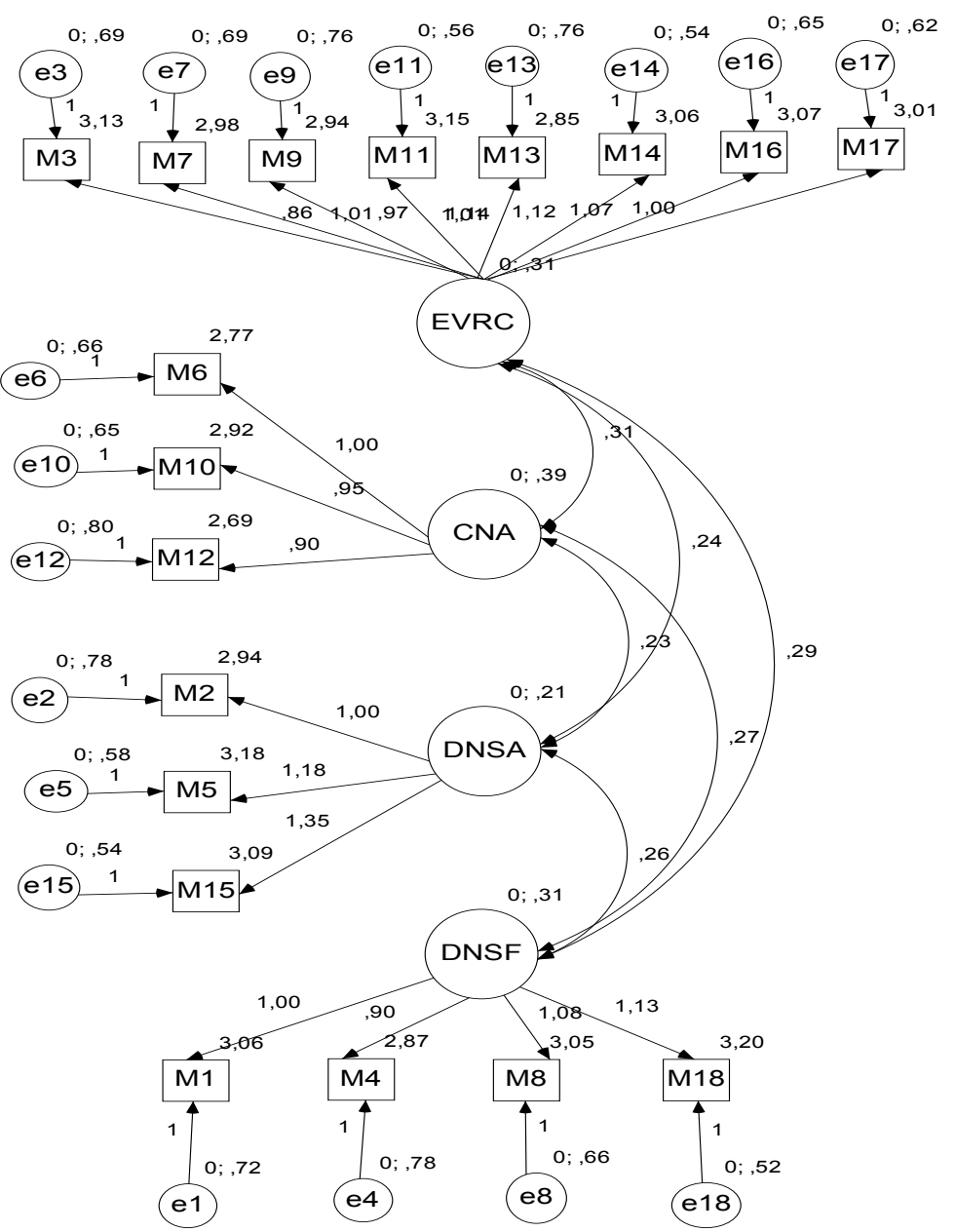

Fuente: elaboración propia a partir de los resultados obtenidos en el AMOS. 
Tabla 3: Estadísticos descriptivos de la frecuencia de comportamientos violentos en el aula 2017-2018

\begin{tabular}{lrr}
\hline Factores & X & DT \\
\hline Disrupción en el aula (DA) & 2.94 & 1.07 \\
Violencia Verbal del Alumnado hacia el Alumnado (VVAA) & 2.79 & 0.98 \\
Violencia Verbal del Alumnado hacia el Profesorado (VVAP) & 2.18 & 0.93 \\
Violencia Física Directa y Amenazas entre Estudiantes (VFDAE) & 2.69 & 0.98 \\
Violencia Física Indirecta por parte del Alumnado (VFIA) & 2.19 & 0.98 \\
Exclusión Social entre Estudiantes (ES) & 2.10 & 0.80 \\
Violencia del Profesorado hacia el Alumnado (VPA) & 1.79 & 0.68 \\
\hline
\end{tabular}

Fuente: elaboración propia a partir de los resultados obtenidos en el análisis de datos.

A continuación, se realiza con estos resultados un análisis diferencial en función de la percepción de la convivencia escolar. Éste se inicia con la variable género (ver Tabla 4), donde los resultados evidencian que, en los cuatro factores considerados, son las mujeres quienes en mayor medida afirman percibir la existencia de normas de convivencia dentro del ámbito escolar; sin embargo, el tamaño de efecto en todos los casos es despreciable.

Tabla 4: Prueba de Mann-Whitney para los factores de convivencia escolar en función del sexo 2017-2018

\begin{tabular}{|c|c|c|c|c|c|c|c|}
\hline Factores & Sexo & $\mathrm{N}$ & $\begin{array}{r}\text { Rango } \\
\text { promedio }\end{array}$ & $\begin{array}{r}\text { U de } \\
\text { Mann-Whitney }\end{array}$ & Z & Sig. & T.E \\
\hline \multirow{2}{*}{ EVRC } & Varones & 928 & 913.97 & \multirow{2}{*}{$417,108.50$} & \multirow{2}{*}{-4.438} & \multirow{2}{*}{0.000} & \multirow{2}{*}{0.10} \\
\hline & Mujeres & 1,017 & $1,026.86$ & & & & \\
\hline \multirow{2}{*}{ CNA } & Varones & 928 & 929.55 & \multirow{2}{*}{$431,569.00$} & \multirow{2}{*}{-3.290} & \multirow{2}{*}{0.001} & \multirow{2}{*}{0.074} \\
\hline & Mujeres & 1,017 & $1,012.65$ & & & & \\
\hline \multirow{2}{*}{ DNSF } & Varones & 928 & 931.24 & \multirow{2}{*}{$433,134.00$} & \multirow{2}{*}{-3.154} & \multirow{2}{*}{0.002} & \multirow{2}{*}{0.071} \\
\hline & Mujeres & 1,017 & $1,011.11$ & & & & \\
\hline \multirow{2}{*}{ DNSA } & Varones & 928 & 922.59 & \multirow{2}{*}{$425,103.00$} & \multirow{2}{*}{-3.829} & \multirow{2}{*}{0.000} & \multirow{2}{*}{0.086} \\
\hline & Mujeres & 1,017 & $1,019.00$ & & & & \\
\hline
\end{tabular}

EVRC: Educación en Valores y Resolución de Conflictos; CNA: Consenso de Normas de Aula; DNSF: Difusión de normas y Sanciones a Familia; DNSA: Difusión de Normas y Sanciones a Alumnado.

Fuente: elaboración propia a partir de los resultados obtenidos en el análisis de datos.

La consideración de la variable diferencial edad ofrece unos resultados diferentes, ya que solamente el factor Consenso de normas de aula refiere 
diferencias estadísticamente significativas; de nuevo el tamaño de efecto es despreciable, en tanto los alumnos de 14 a 17 años son quienes perciben en mayor grado este factor de convivencia $(\mathrm{p}=0.044$; T.E $=0.045)$.

Por otro lado, no afloran diferencias en función del éxito o rendimiento académico del alumno, ya que en todos los casos se obtuvo un nivel de significación mayor a 0.05 . Sin embargo, el curso académico ofrece diferencias en un nivel de significación elevado en la educación de valores y resolución de conflictos, la difusión de normas y sanciones a familia, así como las sanciones al alumnado (en todos los casos, mayor en los que cursan $7^{\circ}$, seguidos de los de curso superior), como se confirma en la Tabla 5.

Tabla 5: Prueba de Kruskal-Wallis para los factores de convivencia escolar en función del curso académico 2017-2018

\begin{tabular}{|c|c|c|c|c|c|}
\hline Factores & $\begin{array}{c}\text { Curso } \\
\text { académico }\end{array}$ & $\mathrm{N}$ & $\begin{array}{l}\text { Rango } \\
\text { promedio }\end{array}$ & $\begin{array}{c}\text { Chi-cuadrado } \\
\text { (Kruskal-Wallis) }\end{array}$ & Sig. \\
\hline \multirow{4}{*}{ EVRC } & $5^{\circ}$ & 143 & 830.79 & \multirow{4}{*}{16.692} & \multirow{4}{*}{0.001} \\
\hline & $6^{\circ}$ & 366 & 944.72 & & \\
\hline & $7^{\circ}$ & 548 & 1034.14 & & \\
\hline & $8^{\circ}$ & 888 & 969.83 & & \\
\hline \multirow{4}{*}{ CNA } & $5^{\circ}$ & 143 & 862.47 & \multirow{4}{*}{6.972} & \multirow{4}{*}{0.073} \\
\hline & $6^{\circ}$ & 366 & 1001.63 & & \\
\hline & $7^{\circ}$ & 548 & 986.87 & & \\
\hline & $8^{\circ}$ & 888 & 970.44 & & \\
\hline \multirow{4}{*}{ DNSF } & $5^{\circ}$ & 143 & 758.87 & \multirow{4}{*}{31.470} & \multirow{4}{*}{0.000} \\
\hline & $6^{\circ}$ & 366 & 920.25 & & \\
\hline & $7^{\circ}$ & 548 & 1031.14 & & \\
\hline & $8^{\circ}$ & 888 & 993.45 & & \\
\hline \multirow{4}{*}{ DNSA } & $5^{\circ}$ & 143 & 739.46 & \multirow{4}{*}{38.104} & \multirow{4}{*}{0.000} \\
\hline & $6^{\circ}$ & 366 & 919.49 & & \\
\hline & $7^{\circ}$ & 548 & 1042.19 & & \\
\hline & $8^{\circ}$ & 888 & 989.96 & & \\
\hline
\end{tabular}

EVRC: Educación en Valores y Resolución de Conflictos; CNA: Consenso de Normas de Aula; DNSF: Difusión de normas y Sanciones a Familia; DNSA: Difusión de Normas y Sanciones a Alumnado.

Fuente: elaboración propia a partir de los resultados obtenidos en el análisis de datos.

Es de resaltar, por otro lado, que la zona urbana donde se sitúa el centro escolar ofrece diferencias en los cuatro factores referidos a la convivencia escolar, siendo los centros situados en zonas más marginales quienes 
perciben un mayor grado de las normas de convivencia o comportamiento escolar; como en los casos anteriores, estas diferencias ofrecen un tamaño de efecto despreciable.

Tabla 6: Prueba de Mann-Whitney para los factores de convivencia escolar en función del tipo de centro 2017-2018

\begin{tabular}{|c|c|c|c|c|c|c|c|}
\hline Factores & Tipo de centro & $\mathrm{N}$ & $\begin{array}{r}\text { Rango } \\
\text { promedio }\end{array}$ & $\begin{array}{r}\text { U de } \\
\text { Mann-Whitney }\end{array}$ & Z & Sig. & T.E \\
\hline \multirow{2}{*}{ EVRC } & Urbano & 890 & 913.70 & \multirow{2}{*}{416696.50} & \multirow{2}{*}{-4.286} & \multirow{2}{*}{0.000} & \multirow{2}{*}{0.097} \\
\hline & Urbano marginal & 1,055 & $1,023.03$ & & & & \\
\hline \multirow{2}{*}{ CNA } & Urbano & 890 & 903.92 & \multirow{2}{*}{407998.00} & \multirow{2}{*}{-5.029} & \multirow{2}{*}{0.000} & \multirow{2}{*}{0.11} \\
\hline & Urbano marginal & 1,055 & $1,031.27$ & & & & \\
\hline \multirow{2}{*}{ DNSF } & Urbano & 890 & 909.96 & \multirow{2}{*}{413373.00} & \multirow{2}{*}{-4.577} & \multirow{2}{*}{0.000} & \multirow{2}{*}{0.10} \\
\hline & Urbano marginal & 1,055 & $1,026.18$ & & & & \\
\hline \multirow{2}{*}{ DNSA } & Urbano & 890 & 914.44 & \multirow{2}{*}{417358.00} & \multirow{2}{*}{-4.276} & \multirow{2}{*}{0.000} & \multirow{2}{*}{0.096} \\
\hline & Urbano marginal & 1,055 & $1,022.40$ & & & & \\
\hline
\end{tabular}

EVRC: Educación en Valores y Resolución de Conflictos; CNA: Consenso de Normas de Aula; DNSF: Difusión de normas y Sanciones a Familia; DNSA: Difusión de Normas y Sanciones a Alumnado. Fuente: elaboración propia a partir de los resultados obtenidos en el análisis de datos.

El análisis de los resultados para establecer la predicción que cada uno de los factores de convivencia tiene en la posible aparición de formas de conductas violentas dentro del contexto escolar, nos permite resaltar algunas particularidades (ver Tabla 7).

En cuanto a la disrupción en el aula, ésta parece estar relacionada con: El consenso de normas de aula, la difusión de normas y sanciones a familia, y la difusión de normas y sanciones a alumnado. En concreto, mientras en los dos últimos casos, es decir, cuando se da un mayor grado de esta difusión de las normas y de sus posibles sanciones, tanto a la familia como al alumnado, aumenta la probabilidad de observar una mayor disrupción en el aula, ocurriría lo contrario cuando se trata del consenso de normas, en tanto parece disminuir en este caso la probabilidad de que se produzca una disrupción. Precisamente este factor de consenso de normas, predice de manera negativa la ocurrencia de todos los factores de violencia escolar, esto es, la formación de dicho consenso disminuye la probabilidad de que sucedan conductas violentas en el aula en sus diferentes manifestaciones, a excepción de la exclusión social (en ella se observa que no existen diferencias). 
Percepción diferencial de la intervención para la convivencia entre escolares de Santo Domingo ... / C. BRINGAS et al.

Tabla 7: Regresión lineal para la predicción de cada uno de los factores de violencia escolar en función de los factores de convivencia escolares 2017-2018

\begin{tabular}{|c|c|c|c|c|c|c|}
\hline & & \multicolumn{2}{|c|}{$\begin{array}{l}\text { Coeficientes no } \\
\text { estandarizados }\end{array}$} & \multirow{2}{*}{$\begin{array}{r}\text { Coeficientes } \\
\text { estandarizados } \\
\text { B }\end{array}$} & \multirow[t]{2}{*}{$\mathrm{t}$} & \multirow[t]{2}{*}{ Sig. } \\
\hline & & B. & Error tip & & & \\
\hline \multirow{4}{*}{ DA } & EVRC & -0.004 & 0.062 & -0.002 & -0.060 & 0.952 \\
\hline & CNA & -0.199 & 0.041 & -0.141 & -4.848 & 0.000 \\
\hline & DNSF & 0.131 & 0.049 & 0.086 & 2.678 & 0.007 \\
\hline & DNSA & 0.163 & 0.048 & 0.107 & 3.368 & 0.001 \\
\hline \multirow{4}{*}{ VVAA } & EVRC & -0.093 & 0.057 & -0.060 & -1.636 & 0.102 \\
\hline & CNA & -0.120 & 0.038 & -0.093 & -3.183 & 0.001 \\
\hline & DNSF & 0.149 & 0.045 & 0.107 & 3.302 & 0.001 \\
\hline & DNSA & 0.053 & 0.045 & 0.038 & 1.194 & 0.233 \\
\hline \multirow{4}{*}{ VVAP } & EVRC & -0.147 & 0.054 & -0.100 & -2.742 & 0.006 \\
\hline & CNA & -0.099 & 0.036 & -0.081 & -2.772 & 0.006 \\
\hline & DNSF & 0.071 & 0.043 & 0.054 & 1.662 & 0.097 \\
\hline & DNSA & -0.003 & 0.042 & -0.002 & -0.069 & 0.945 \\
\hline \multirow{4}{*}{ VFDAE } & EVRC & -0.066 & 0.057 & -0.042 & -1.160 & 0.246 \\
\hline & CNA & -0.126 & 0.038 & -0.098 & -3.333 & 0.001 \\
\hline & DNSF & 0.061 & 0.045 & 0.043 & 1.341 & 0.180 \\
\hline & DNSA & 0.001 & 0.045 & 0.001 & 0.027 & 0.979 \\
\hline \multirow{4}{*}{ VFIA } & EVRC & -0.092 & 0.050 & -0.067 & -1.832 & 0.067 \\
\hline & CNA & -0.085 & 0.034 & -0.074 & -2.541 & 0.011 \\
\hline & DNSF & -0.011 & 0.040 & -0.009 & -0.279 & 0.781 \\
\hline & DNSA & 0.005 & 0.040 & 0.004 & 0.128 & 0.898 \\
\hline \multirow{4}{*}{ ES } & EVRC & -0.033 & 0.047 & -0.026 & -0.717 & 0.473 \\
\hline & CNA & -0.050 & 0.031 & -0.048 & -1.619 & 0.106 \\
\hline & DNSF & 0.003 & 0.037 & 0.002 & 0.069 & 0.945 \\
\hline & DNSA & -0.053 & 0.037 & -0.046 & -1.441 & 0.150 \\
\hline \multirow{4}{*}{ VPA } & EVRC & -0.145 & 0.039 & -0.134 & -3.750 & 0.000 \\
\hline & CNA & -0.106 & 0.026 & -0.118 & -4.117 & 0.000 \\
\hline & DNSF & 0.022 & 0.031 & 0.023 & 0.729 & 0.466 \\
\hline & DNSA & -0.036 & 0.030 & -0.037 & -1.183 & 0.237 \\
\hline \multicolumn{7}{|c|}{$\begin{array}{l}\text { DA: Disrupción en el aula; VVAA: Violencia Verbal del Alumnado hacia el Alumnado; VVAP: } \\
\text { Violencia Verbal del Alumnado hacia el Profesorado; VFDAE: Violencia Física Directa y Amenazas } \\
\text { entre Estudiantes; VFIA: Violencia Física Indirecta por parte del Alumnado; ES: Exclusión Social } \\
\text { entre Estudiantes; VPA: Violencia del Profesorado hacia el Alumnado } \\
\text { EVRC: Educación en Valores y Resolución de Conflictos; CNA: Consenso de Normas de Aula; } \\
\text { DNSF: Difusión de normas y Sanciones a Familia; DNSA: Difusión de Normas y Sanciones a } \\
\text { Alumnado. }\end{array}$} \\
\hline
\end{tabular}


La difusión de normas y sanciones a familia también se relaciona con la probabilidad de que se produzca la violencia verbal entre el alumnado, mientras que la educación en valores y resolución de conflictos parece afectar a la violencia verbal hacia el profesorado por parte de los alumnos, así como la violencia del profesorado hacia el alumnado; en ambos casos, estas medidas de convivencia disminuyen las posibilidades de que se den ambas formas de violencia

\section{DisCUSIÓN Y CONCLUSIONES}

Los resultados de nuestro estudio han puesto de manifiesto que se cuenta con un instrumento que evalúa la percepción del alumnado en Educación Primaria de las medidas de convivencia desarrolladas en el marco escolar de la República Dominicana. El análisis factorial confirmatorio, de acuerdo a lo esperado (Álvarez-García et al., 2012), presenta este constructo con un ajuste satisfactorio de los datos para la muestra de la comunidad escolar de República Dominicana. Si bien hay que reconocer que los índices de fiabilidad de cada uno de los factores que lo componen, son satisfactorios, aunque no elevados (amplitud de rango del Coeficiente $\alpha$ de Cronbach en los factores entre 0.57 a 0.79 ), también hay que señalar que el número de items de cada uno de los factores son más bien reducidos. De hecho, en la población española los resultados han sido similares a los obtenidos en nuestra adaptación ( $\alpha$ de Cronbach en el rango entre 0.63 a 0.78 ).

Por otra parte, nuestro estudio ofrece la percepción que los estudiantes de centros educativos de Santo Domingo (República Dominicana) tienen de las normas implementadas para la mejora de la convivencia escolar y su relación con la frecuencia que ellos refieren de dicha violencia en el marco educativo. Sin embargo, hay que reconocer la necesidad de conocer, aunque de forma generalizada, las conductas violentas y su problemática en las aulas de los centros educativos, con el objeto de comprender si esa percepción responde a una necesidad de instaurar normas de mejora de la convivencia, tal como proponen Álvarez-García, Núñez, y Dobarro (2013). De entrada, nuestros resultados constatan que la violencia en las aulas es un hecho que continúa presente en los centros de Santo Domingo, especialmente en lo concerniente a las conductas disruptivas dentro del aula, así como las diferentes manifestaciones físicas, verbales y psicológicas que ocurren entre los propios estudiantes. Estos resultados están en la línea de los comportamientos que refieren diferentes estudios en otras realidades contextuales (Eljach, 2011; Román, y Murillo, 2011; Álvarez-García et al., 2012; Mercedes, 2016; Smith, y López-Castro, 2017; Postigo et al., 2019). 
Así, en relación a la sensación que muestran sobre la incorporación de pautas comportamentales que marcan una adecuada convivencia dentro de las aulas, queda constatada (aunque considerado un margen extenso en la edad de nuestra muestra) que un mayor grado cronológico-evolutivo no mejora sustancialmente en un reconocimiento de mejora de normas de convivencia; solamente los de mayor edad refieren la existencia de un consenso de normas dentro del aula. Llama la atención que, en relación al grado académico, que consideramos se relaciona con la edad, este factor no ofrece ningún resultado a considerar, mientras que por el contrario los otros tres (educación en valores y resolución de conflictos, difusión de normas y sanciones, tanto a la familia como al alumnado) son los de los dos últimos grados quienes aseguran la existencia de dichas pautas de comportamiento. De igual modo, las alumnas observan que se establecen las mejoras necesarias para aumentar la calidad de la convivencia escolar, mientras que la variable referida al rendimiento académico no se relaciona con una diferenciación en la percepción de normas de convivencia. El tipo de centro educativo, sin embargo, sí se relaciona con este último factor, siendo los estudiantes de los centros procedentes de zonas más marginales, y donde se supone que mayor es el conflicto, los que consideran que se cumplen cada uno de los factores que mejoran la convivencia entre los alumnos (Álvarez-García, Dobarro, Rodríguez, Núñez y Álvarez, 2013; Yeager, Fong, Lee and Espelage, 2015).

Finalmente, hemos querido conocer si el establecimiento de unas normas mínimas de convivencia podrían determinar la aparición o, mejor aún, la eliminación de determinados comportamientos reprobables en los jóvenes escolares. Pues bien, de acuerdo con Álvarez-García, Dobarro, Rodríguez, Núñez y Álvarez (2013) y salvo para el caso de la exclusión social, el comportamiento conflictivo dentro del aula en sus diferentes manifestaciones (violencia física y verbal entre los propios estudiantes, y hacia el profesorado, así como la ejercida por éste último) disminuye su probabilidad de aparición ante una propuesta de normas o pautas conductuales aprobadas por la comunidad educativa. No obstante, la publicidad de estas normas, junto con las sanciones en casos de incumplimiento, especialmente a sus familias (generalmente los progenitores), provocan el efecto contrario, ya que tanto las conductas disruptivas en el aula como la violencia verbal ejercida por el alumno aumentan ante este factor. Sucede lo mismo cuando se trata de informar de las consecuencias en el alumnado de la violación de las normas de conducta. Por lo demás, la educación en valores y resolución de conflictos desempeña el mismo rol que el establecimiento de normas 
en el aula, pues reduce significativamente los comportamientos agresivos relacionados con la violencia verbal del estudiante hacia el profesor, así como la de este último a sus alumnos.

Estos resultados nos acercan a la idea de que una propuesta de pautas comportamentales, que suponemos, será no solamente aceptada por el equipo directivo del centro educativo, sino también por todos los integrantes de la comunidad escolar, junto con la enseñanza de valores y formas de resolver problemas, resulta más positivo que la mera "amenaza" a quienes tratan de alterar la buena convivencia en el centro. Por tanto, y aproximándonos a los estudios de López-Latorre et al. (2002), Dick, y Ferguson (2015) o Peñalva-Vélez et al. (2015), nuestros resultados apoyan que resulta más sano incitar a realizar conductas positivas que informar de los efectos del comportamiento negativo para eliminar este último. Es decir, más que el uso del castigo como corrección de conductas no deseables al no tener una eficacia demostrada a largo plazo (Yeager, Fong, Lee and Espelage, 2015), reiteramos nuestra convicción del papel de la sociedad y de las instancias socializadoras en la enseñanza de medidas de protección y de educación en valores positivos. Ello implica el instaurar normas prosociales de comportamiento incompatibles con aquellas conductas que deseamos, no lleguen a producirse (Cutrín, Gómez-Fraguela, Maneiro y Sobral, 2017; Ruiz-Hernández, Moral-Zafra, Llor-Esteban, y Jiménez-Barbero, 2019).

Asumimos que estos resultados ofrecen limitaciones. Aceptamos que, al estar valorando la relación entre la percepción de las normas de convivencia entre los alumnos en relación con la violencia, basándonos en diferentes características personales y situacionales, sería interesante contar con un colectivo estudiantil con características comportamentales violentas, incluso que hubiesen sido (aunque no necesariamente) sancionados en algún momento. Esta variable sería de interés, en tanto hubiese permitido comprobar las diferencias existentes de la instauración de pautas de sociabilidad según sus propias normas comportamentales, tanto prosociales como violentas, en la percepción que tienen los chicos y chicas jóvenes de Santo Domingo. Si bien es cierto que el colectivo que hemos utilizado en nuestro estudio, procedente de zonas urbanas y zonas urbanas-marginales, nos acerca a nuestro objetivo, no pueden tomarse los resultados como consistentes para resolver realidades de conflicto en las aulas. Esta afirmación podríamos sostenerla en tanto las diferencias se dan más por niveles económicos que por el comportamiento en sí. 
Percepción diferencial de la intervención para la convivencia entre escolares de Santo Domingo ... / C. BRINGAS et al.

\section{REFERENCIAS BIBLIOGRÁFICAS}

Alarcón, P.A., Pérez-Luco, R., Wenger, L., Salvo, S., y Chesta, S., 2018, "Personalidad y gravedad delictiva en adolescentes con conducta antisocial persistente", en Revista Iberoamericana de Psicología y Salud, 9(1), 58-74, doi: https://doi. org/10.23923/j.rips.2018.01.015

Álvarez-García, D., Dobarro, A., Álvarez-Pérez, L., Núñez, C., y Rodríguez, C., 2014, "La violencia escolar en los centros de educación secundaria de Asturias desde la perspectiva del alumnado" en Educación, XX1: Revista de la Facultad de Educación, 17(2), 337-360, doi: 10.5944/educxx1.17.1.11494

Álvarez-García, D., Dobarro, A., Rodríguez, C., Núñez, C., y Álvarez, L., 2013, "Consensus on classroom rules and its relationship with low levels of school violence", en Infancia y Aprendizaje, 36(2), 199-217, doi: https://doi. org/10.1174/021037013806196229

Álvarez-García, D., Mercedes, J., Rodríguez, F.J., y Núñez, C., 2015, “Adaptación y validación del cuestionario $\mathrm{CUVE}^{3}$-EP para la evaluación de la violencia escolar en centros de enseñanza básica de la República Dominicana", en Anales de Psicología, 31(3), 859-868, doi: http://dx.doi.org/10.6018/analesps.31.3.186482

Álvarez-García, D., Núñez, C., Dobarro, A., y Rodríguez, C., 2012, "Propiedades psicométricas del Cuestionario de Medidas para la Mejora de la Convivencia en Educación Primaria (Cuestionario M-EP)", en Revista Electrónica de Metodología Aplicada, 17(2), 1-17

Álvarez-García, D., Núñez, C., y Dobarro, A., 2013, “Cuestionarios para evaluar la violencia escolar en Educación Primaria y en Educación Secundaria: CUVE ${ }^{3}$-EP y CUVE ${ }^{3}$-ESO”, en Apuntes de Psicología, 31(2), 191-202

APA, 2009, Publication Manual of the American Psychological Association (6th ed.). Washington, DC American Psychological Association

Borowsky, I.W., Taliaferro, L.A., and McMorris, B.J., 2013, "Suicidal thinking and behavior among youth involved in verbal and social bullying: risk and protective factors", in Journal of Adolescent. Health, 53 (Suppl), S4-12, doi: 10.1016/j. jadohealth.2012.10.280.

Cohen, J., 1988, Statistical power analysis for the behavioural sciences. (2 $2^{\mathrm{a}}$. ed). NJ EEUU Erlbaum

Compton, L., Campbell, M. A., and Mergler, A., 2014, "Teacher, parent and student perceptions of the motives of cyberbullies", in Social Psychology of Education, 17(3), 383-400, doi:10.1007/s11218-014-9254-x

Cutrín, O., Gómez-Fraguela, J.A., Maneiro, L., y Sobral, J., 2017, “Effects of parenting practices through deviant peers on non violent and violent antisocial behaviours in middle- and late-adolescence", in The European Journal of Psychology Applied to Legal Context, 9, 75-82, doi: http://dx.doi.org/10.1016/j. ejpal.2017.02.001 
Dick, B., and Ferguson, B.J., 2015, "Health for the world's adolescents: a second chance in the second decade", in Journal of Adolescent Health, 56(1), 3-6.

Eljach, S., 2011, Violencia escolar en América Latina y el Caribe Superficie y fondo. Panamá Unicef.

Hymel, S., and Swearer, S. M., 2015, "Four decades of research on school bullying: An introduction", in American Psychologist, 70(4), 293-299, doi:10.1037/ a0038928

López-Latorre, MJ., Garrido, V., Rodríguez, F.J., y Paíno, S., 2002, “Jóvenes y competencia social: Un programa de intervención”, Psicothema, 14 (Supl), 155163

McGee, Z.T., Logan, K., Samuel, J., and Nunn, T., 2017, “A multivariate analysis of gun violence among urban youth: The impact of direct victimization, indirect victimization, and victimization among peers", in Cogent Social Sciences, 3(1), 1-8, doi: 10.1080/23311886.2017.1328772

Méndez, I., y Cerezo, F., 2018, "La repetición escolar en educación secundaria y factores de riesgo asociados", en Educación XX1, 21(1), 41-62, doi: 10.5944/ educXX1.20172

Mercedes, J., 2016, Evaluación de la convivencia escolar en los centros educativos de Santo Domingo (República Dominicana). Tesis Doctoral no publicada, Oviedo Universidad de Oviedo

Novo, M., Herbón, J., y Amado, B.G., 2016, “Género y victimización: efectos en la evaluación de la violencia psicológica sutil y manifiesta, apego adulto y tácticas de resolución de conflictos", en Revista Iberoamericana de Psicología y Salud, 7, 89-97, doi: http://dx.doi.org/10.1016/j.rips.2016.05.002

Olweus, D., 2012, “Cyberbullying: An overrated phenomenon?”, in European Journal of Developmental Psychology, 9, 520-538, doi: https://doi.org/10.1080/ 17405629.2012 .682358

Pacheco-Salazar, B., 2018, "Violencia Escolar: la perspectiva de estudiantes y docentes", en REDIE, 20 (1), 112-121. doi: https://doi.org/10.24320/redie.2018.20.1.1523

Pacheco-Salazar, B., y Hernández-Valerio, W., 2014, "La convivencia en los centros educativos de educación básica de República Dominicana”, en Gairín-Sallán, Joaquín y Barrera-Corominas, Aleix (Coord.). La convivencia en los centros educativos de educación básica en Iberoamérica. Santiago de Chile: Santillana.

Peñalva-Vélez, A., López-Goñi, J. J., Vega-Osés, A., y Satrústegui-Azpíroz, C., 2015, "Clima escolar y percepciones del profesorado tras la implementación de un programa de convivencia escolar", en ESE. Estudios sobre Educación, 28, 9-28, doi: https://doi.org/10.15581/004.28.9-28

Pérez-Fuentes, M.C., Gázquez, J.J., Fernández-Baena, R.J., y Molero, M.M., 2011, "Análisis de las publicaciones sobre convivencia escolar en una muestra de revistas de Educación en la última década”, en Aula Abierta, 39(2), 81-90. 
Postigo, S., González, R., Montoya, I., y Ordóñez, A., 2013, "Propuestas teóricas en la investigación del acoso escolar: Una revisión", en Anales de Psicología, 29(2), 413-425, doi:10.6018/analesps.29.2.148251

Postigo, S., Schoeps, K., Ordóñez, A., y Montoya-Castilla, I., 2019, “¿Qué dicen los adolescentes sobre el acoso escolar?”, en Anales de Psicología, 35(2), 251258, doi: http://dx.doi.org/10.6018/analesps.35.2.301201

Román, M., y Murillo, F.J., 2011, “América Latina: violencia entre estudiantes y desempeño escolar”, en Revista CEPAL, 104, 37-54.

Ruiz-Hernández, J.A., Moral-Zafra, E., Llor-Esteban, B., y Jiménez-Barbero, J.A., 2019, "Influence of Parental Styles and Other Psychosocial Variables on the Development of Externalizing Behaviors in Adolescents: A Sytematic Review", en The European Journal of Psychology Applied to Legal Context, 11(1), 9-21, doi: https://doi.org/10.5093/ejpalc2018a11

Smith, P.K., and López-Castro, L., 2017, "Cross-National Data on Victims of Bullying: How does PISA Measure up with Other Surveys? An Update and Extension of the Study by Smith, Robinson, and Marchi (2016)", in International Journal of Developmental Sciences, 11(3-4), 87-92, doi: 10.3233/DEV-170227

Smith, P.K., and Robinson, S., 2019, "How Does Individualism-Collectivism Relate to Bullying Victimisation?", in International Journal of Bullying Prevention, 1, 3-13, https://doi.org/10.1007/s42380-018-0005-y

Vargas, T., 2010, Violencia en la escuela. Estudio Cualitativo 2008-2009. Santo Domingo: Plan República Dominicana.

Veenstra, R., Lindenberg, S., Huitsing, G., Sainio, M., and Salmivalli, C., 2014, "The role of teachers in bullying: The relation between antibullying attitudes, efficacy, and efforts to reduce bullying", in Journal of Educational Psychology, 106(4), 1135-1143, doi: http://doi.org/10.1037/a0036110

Wasserstein, R.L. and Lazar, N.A., 2016, “The ASA's statement on p-values: context, process, and purpose", in The American Statistician, 70(2), 129-133, doi: https://doi.org/10.1080/00031305.2016.1154108

Yeager, D.S., Fong, C.J., Lee, H.Y., and Espelage, D.L., 2015, "Declines in efficacy of anti-bullying programs among older adolescents: Theory and a three-level meta-analysis", in Journal of Applied Developmental Psychology, 37, 36-51, doi: https://doi.org/10.1016/j.appdev.2014.11.005

\section{RESUMEN CURRICULAR DE LAS AUTORAS Y DE LOS AUTORES}

\section{Carolina Bringas Molleda}

Profesora Contratada Doctora del Departamento de Psicología y Antropología de la Universidad de Extremadura (España), dentro del área Psicología Evolutiva y de la Educación. Ha publicado diversos artículos en revistas científicas, y capítulos de libro, así como contribuido a diversos congresos 
nacionales e internacionales. También ha participado como miembro investigador en proyectos de investigación financiados por diversos organismos. Es miembro del Grupo de Investigación Psicología Educativa, Social y de la Personalidad (GIPES) de la Universidad de Extremadura, y coordinadora del Grupo de Innovación Docente Inclusión, Aprendizaje y Salud. Actualmente cuenta con un sexenio de investigación.

Dirección electrónica: cbringas@unex.es

Registro ORCID: https://orcid.org/0000-0001-7956-2337

Josefina Mercedes Acosta

Profesora de la Universidad Autónoma de Santo Domingo. Doctora por la Universidad de Oviedo, España. Título de Profesora Meritísima de la Facultad de Ciencias de la Educación de la Universidad Autónoma de Santo Domingo. Publicación de la obra Aprendizaje Participativo de las Ciencias Naturales, Premio anual de Didáctica, Manuel Peña y Reynoso, República Dominicana. Publicación de artículos en revistas científicas. En la actualidad consultora nacional de formación docente del Ministerio de Educación Superior Ciencia y Tecnología de República Dominicana. Fue miembro del equipo rediseño curricular Facultad de Humanidades, UASD y del equipo de evaluación de profesores para la transferencia de categoría de adjunto a titular de la Facultad de Humanidades, UASD. Fue Directora de Investigación, Extensión y Publicaciones del Instituto Superior de Formación Docente Salomé Ureña y directora académica de esa institución. Dirección electrónica: josefinamer11@yahoo.com

Registro ORCID: https://orcid.org/0000-0003-2129-9593

\section{David Álvarez García}

Profesor titular en el área de Psicología Evolutiva y de la Educación de la Universidad de Oviedo (España). Miembro del grupo de investigación en Aprendizaje Escolar, Dificultades y Rendimiento Academico (ADIR). Ha participado en proyectos de investigación como "Análisis de la convivencia escolar en los centros educativos del Principado de Asturias", "Factores de riesgo asociados a cibervíctimización en la adolescencia" o "La formación de los futuros docentes frente a la violencia escolar". En la actualidad es investigador principal del proyecto "Claves para la prevención del rechazo y el acoso escolar entre estudiantes en Educación Primaria", financiado por la Agencia Estatal de Investigación del Gobierno de España. Es coautor de instrumentos para la evaluación de la convivencia escolar, como los cuestionarios CUVE3 (Ed. Albor-Cohs, 2012), y de la ciberagre- 
sión entre estudiantes, como los cuestionarios CYVIC y CYBA; así como del programa de intervención Aprende a Resolver Conflictos (ARCO) (Ed. CEPE, 2007).

Dirección electrónica: alvarezgardavid@uniovi.es

Registro ORCID: https://orcid.org/0000-0002-7460-497X

\section{Margarita Almonte Mata}

Profesora adjunta en la escuela de Orientación y Psicopedagogía Universidad Autónoma de Santo Domingo (UASD). Licenciada en Orientación Educativa, Especialidad en Orientación Educativa e intervención Psicopedagógica (UASD) Maestría en Gerencia y Liderazgo Educativo-Universidad Nova Southeastern, es actualmente Profesora de cuarto nivel de la UASD. Es importante destacar el trabajo en proyectos sociales y como resultados coautora del Manual de Capacitación en Educación Multicultural del Servicio Jesuita de refugiados y Migrantes, autora del artículo Violencia vecinal desde el contexto escolar: bandas, naciones y otros grupos. Fascículo "Yo Evito la Violencia y Tú"- Plan Lea, Listín Diario. Asesora de investigaciones nacionales. Coordinadora del Diplomado Educación y Género del Ministerio de Educación, Plan Internacional y varias universidades nacionales. Cabe destacar el rol de par facilitador en el proyecto de formación a directores de centro educativos con el INAFOCAM y la Universidad de Barcelona.

Dirección electrónica: m.almontem@gmail.com

Registro ORCID: https://orcid.org/0000-0002-8028-1046

Francisco Javier Rodríguez Díaz

Catedrático de Personalidad, Evaluación y Tratamiento Psicológico de la Universidad de Oviedo (España) y Profesor visitante de la Universidad Andres Bello (Chile). Es Doctor en Psicología de la Universidad de Santiago de Compostela desde 1986, resultando ser evaluado positivamente de cinco sexenios de investigación (1987-2020). La trayectoria científica se encuentra en la línea de Psicología Jurídica y Forense, con continuidad de investigación $\mathrm{I}+\mathrm{D}+\mathrm{i}$ hasta la fecha de hoy. Pertenece al Grupo de Investigación CJS (Comunitaria, Jurídica y de la Salud) desarrollo del GIP (Grupo de Investigación Psicosocial). Autor de cerca de 100 artículos publicados en revistas ISI, entre ellas, Psicothema, Salud Pública de México, Journal of Interpersonal Violence, The European Journal of Psychology Applied to Legal Context, Violence Against Women, Psychology of Violence, Frontiers, Plos One, entre otras. Ha sido director de una línea doctoral 
y de una veintena de tesis doctorales, publicando diversos libros en editoriales prestigiosas como Manual moderno.

Dirección electrónica: franciscojavierrodriguezdiaz@gmail.com

Registro ORCID: http://orcid.org/0000-0002-5899-439x

Artículo recibido el 13 de abril de 2020 y aprobado el 15 de julio de 2021. 